\title{
General Guide for Korean Acupuncture \& Moxibustion
}

\author{
Hyo-Jung Kwon, Yong-Suk Kim* \\ Department of Acupuncture \& Moxibustion, (Brain \& Neurological Disorders and Pain), Kangnam Korean \\ Hospital, Kyung Hee University, Seoul, Korea \\ Email: ackys@hanmail.net
}

Received 14 May 2015; accepted 25 August 2015; published 28 August 2015

Copyright (C) 2015 by authors and Scientific Research Publishing Inc.

This work is licensed under the Creative Commons Attribution International License (CC BY). http://creativecommons.org/licenses/by/4.0/

(c) (i) Open Access

\section{Abstract}

As ancient Korean and Chinese dynasties held close relationships in politics and culture throughout history, the medicine of the two nations were developed in mutual influence. For instance, the current version of Lingshu, the second half of the Huangdineijing, was transcribed from The Bible of Acupuncture, which was brought to the Chinese Song dynasty from the Korean Koryo dynasty in 1092. While maintained a close relationship with traditional Chinese medicine, Korean medicine had continued to develop unique systems on its own over the history and established typical types of acupuncture methods, which are different from those of traditional Chinese medicine [1]. In the 17th century, a royal physician by the name of Dr. Heo Jun wrote Donguibogam, the first encyclopedia of Korean medicine in Korea. It remained to be a book of instruction for Korean Medicine Doctors. Another book called Donguisusebowon was published in 1901 on the basis of the theory of four constitutions by Dr. Lee Je-Ma at the end of the Chosun dynasty [2]. There were two other representative Korean acupuncture methods: Saam acupuncture and Constitutional acupuncture (Taeguek acupuncture). Theories of Korean acupuncture applied a summarized framework for each individual to diagnose a physical condition, explaining the biologic phenomena within the concept of constitutional medicine [1]. The diagnosis systems of several Korean acupuncture styles were focused on simplifying the understanding of the body's core imbalances, and the resulting diagnosis enabled practitioners to devise therapeutic strategies that were based on constitutional energy traits. Saam acupuncture used 12 energy (or Qi) traits, mainly controlled by the 12 corresponding Meridians, that underlie diverse superficial biologic phenomena [2] [3]. It was suggested that these 12 energies determined the inclinations of the whole body, and they were targeted to recover the balance of the body's constancy. Taegeuk acupuncture was identified by Sasang constitutional medicine according to the patient's innate constitution. Sasang constitutional medicine identified four constitutions according to the individual's inherent disparities among major Organ energies, expressed as the size of the Organs, all of which determined the physiologic characteristics of the individual patient and served as a major imbalance succeeding diverse pa-

*Corresponding author. 
thologic processes. These constitutional traits were suggested to be the source of individual differences in apparently similar physiologic or pathologic reactions [2]. As Meridian theory is based on the Qi thesis of Yin-Yang and Five Elements among Organs, acupuncture treatment couldn't be separated from these viewpoints. Saam acupuncture was based on the control of $Q i$ and Blood among Organs and channels, and thus the clinical use of Saam acupuncture treatments seemed to be core to oriental medicine [4]. As the creation of Blood originates from Qi, one could argue that $Q i$ included Blood, thereby stating that the effect of Saam acupuncture was achieved by harmonizing the function and flow of $Q i$. The treatment protocol of Saam acupuncture mainly focused on tonification and sedation of the Five Shu points ("five transport points"), firmly based on regular pattern. It had a strong effect on imbalances of the Five Organs, but on the other hand could be said to have little effect on disease of interruption such as stagnation and irregularities in Meridian networks. Thus, acupoints other than the Five Shu points were used as well.

\section{Keywords}

\section{Saam Acupuncture, Constitutional Acupuncture, Five Elements, Taegeuk Acupuncture}

\section{Introduction}

Saam acupuncture is one of unique Korean acupuncture styles and its technique is widely adopted by clinicians and educational institutions in Korea today. It originated in the 17th century, probably by a Buddhist monk called "Saam". Saam acupuncture provides basic acupuncture prescriptions for the imbalances of each of the twelve Meridians. The main energy traits of the 12 Meridians are employed by applying the promotion cycle (Sheng, creation, "nourishing") and control cycle (Ke, governor, "suppressing") relationships of the Five Elements (or "Five Phases") theory onto the Five Shu points and 12 Meridians [3] [4]. Saam acupuncture summarizes diverse physiological or pathologic processes into 12 images of Qi imbalance corresponding to the $12 \mathrm{Me}-$ ridians and expands the use of the Five Shu points. The manuscripts of Saam acupuncture carry clinical applications and modifications of the basic acupuncture prescriptions [5].

Five Elements acupuncture consists of tonification and sedation using Five Shu points. Five Elements acupuncture, also known as "tonification and sedation in self-Meridian" on the basis of the creation cycle, was first proposed by Gao-wu during the Ming dynasty in China. Saam developed this technique further by extending it into another Meridian and using the governor cycle in self- and other-Meridians.

Gao-Wu, during the Ming Dynasty (1519 AD), was the first and foremost medical acupuncturist to tonify deficiency and sedate the excess on the basis of either depletion or repletion of the promotion cycle, which is explained in "The Four Needle method" by Ross [6] [7]. Saam acupuncture [8] proposes the Five Elements acupuncture style that simultaneously uses the Five Shu points of the promotion and the control cycles, called the "Four Needle technique" by Hicks et al. [9] and the "eight Needle methods" by Ross [6].

Gao-Wu described the use of "tonification and sedation points" along the self Meridian using the Five Shu points. The basis of Gao-Wu's treatment was the creation (mother-child) cycle and is included in the "Four Needle method" with the following rules: to determine the tonification point for a Meridian, select the Mother Element on the Meridian in question, and to determine the sedation points, select the Child Element. But Saam acupuncture, on the basis of Gao-Wu's treatment, created a Five Element acupuncture therapy by adding the control (grandmother-grandchild) cycle, as well as selecting acupuncture points among other related Meridians. From the above approach, this theory consisted of two tonifications and two sedation points, which were selected from among the Five Shu points. The Five Shu points had characteristics of their particular Five Elements theory according to the difficult issues in Nan-Ching.

The principles of Saam acupuncture originated from the creation and controlled cycles among the Five Elements and had the following rules: to determine the tonification and sedation points for a Meridian in deficiencies, first tonified the mother Element Five Shu points on the Meridian in question as well as the master point (Five Shu points of same Element as the Meridian) on the mother Element Meridian, and then sedated the Controller Element Five Shu points on the affected Meridian as well as the master point on the Controller Element 
Meridian; to determine the tonification and sedation points for a Meridian in excesses, first sedated the child Element Five Shu points on the affected Meridian, and then tonified the Controller Element Five Shu points on the affected Meridian as well as the master point on the Controller Meridian.

There also exists another setting of tonification and sedation system called the "coldness-heat acupuncture treatment", derived from the "deficiency-excesses acupuncture treatment", that is simple and rarely used because it selects only the Water and Fire Element Five Shu points among a Meridian and has the following rules: for cold symptoms, to determine the tonification and sedation points for a Meridian, first tonify the Fire Element Five Shu points on the affected Meridian as well as the Fire point on the Fire Meridian-namely HT8 for Yin Meridians and SI5 for Yang Meridians, and then sedate the Water Element Five Shu points on the affected Meridian as well as the Water point on the Water Meridian-namely KI10 for Yin Meridians and BL66 for Yang Meridians.

For heat symptoms, to determine the tonification and sedation points for a Meridian, first sedate the Fire Element Five Shu points on the affected Meridian as well as the Fire point on the Fire Meridian, and secondly tonify the Water Element Five Shu points on the affected Meridian as well as the Water point on the Water Meridian.

\subsection{Acupoints Used in Saam Acupuncture}

The Five Shu points, located below the elbow and knee joints, could be used corresponding to the Five Elements for imbalances in correlation to tonification and sedation [10] (Table 1). Every acupuncture point has an effect on disturbances of the corresponding channel and the coupled channel as well as on the illness of the corresponding Organ, the allocated tissues and sensory Organs [11]. Acupuncture points have an effect on the related channel axis, for example, ST38 along the Yangming Meridian on the shoulder, more namely the Stomach Meridian and the Large Intestine Meridian.

Giovanni [12]: "The sections of channel between fingers/toes and elbows/knees are more superficial than the rest, which is one of the reasons for the importance of the points lying along its path. The energetic action of the points along a section of such a channel is much more dynamic than other points, which explains their frequent use in clinical practice. Another reason for the dynamism of these points is that, at the tips of fingers and toes, the energy changes polarity from Yin to Yang or vice versa and, due to this polarity change, the Qi of the channel is more unstable and thus more easily influenced. The progression of the Five Element points along the channel is probably in relation to this change of polarity, as the second point belongs to the Fire in the Yin channels and the Water in the Yang channels. Five points situated along this channel section are particularly important and are

Table 1. Five Shu Points.

\begin{tabular}{|c|c|c|c|c|c|}
\hline Yin Meridian & Wood (well) & Fire (spring) & Earth (stream) & Metal (river) & Water (sea) \\
\hline Lung (Metal) & LU11 & LU10 & LU9 & LU8 & LU5 \\
\hline Heart (Fire) & HT9 & HT8 & HT7 & HT4 & HT3 \\
\hline Pericardium (Fire) & PC9 & PC8 & PC7 & PC5 & PC3 \\
\hline Spleen (Earth) & SP1 & SP2 & SP3 & SP5 & SP9 \\
\hline Kidney (Water) & KI1 & $\mathrm{KI} 2$ & KI3 & KI7 & KI10 \\
\hline Liver (Wood) & LR1 & LR2 & LR3 & LR4 & LR8 \\
\hline Yang Meridian & Metal (well) & Water (spring) & Wood (stream) & Fire (river) & Earth (sea) \\
\hline Large Intestine (Metal) & LI1 & LI2 & LI3 & LI5 & LI11 \\
\hline Small Intestine (Fire) & SI1 & $\mathrm{SI} 2$ & SI3 & SI5 & SI8 \\
\hline Triple Energizer (Fire) & TE1 & TE2 & TE3 & TE6 & TE10 \\
\hline Stomach (Earth) & ST45 & ST44 & ST43 & ST41 & ST36 \\
\hline Bladder (Water) & BL67 & BL66 & BL65 & BL60 & BL54 \\
\hline Gall Bladder (Wood) & GB44 & GB43 & GB41 & GB38 & GB34 \\
\hline
\end{tabular}


called the Five Transporting points; they also coincide with what are called the Elements points. However, the dynamic of these points is irrespective of their Five Elements character".

Yun-Tao-Ma [13]: "An interesting neurological fact is that the limbs below the elbows and knees occupy large regions in the brain sensory gyrus. Thus, the acupoints below the elbows and knees also occupy a large region in the cortical representation of the brain's post-central sensory gyrus. This correlation may explain the reason why the acupoints below the elbows and knees contain more sensory receptors and needling stimulation of these points can induce a greater reaction and activity in the brain. This principle clearly supports the concept of using certain acupoints below the elbows and knees as diagnosis and treatment points during acupuncture treatment”.

Most illnesses are caused by interruptions of Qi and blood manifested by stagnation and irregularities. Illnesses are also caused by imbalances of Organs brought on by deficiency and excess through the Meridian network. Acupuncture treatments, therefore, must cover these two aspects of illness to achieve acupoint efficacy and functions of tonification and sedation. In order to gain greater efficacy, acupuncture based on acupoint efficacy and Saam acupuncture can be combined to simultaneously correct interruptions and imbalances as well as have tonification-sedation effects. In relations to the principles of acupuncture treatments, the acupuncture procedures based on acupoint efficacy to correct interruptions can be assumed as "branch treatment", and acupuncture based on tonification-sedation effects to correct imbalances can be assumed as "root treatment". Effective acupuncture treatment, therefore, is a combination of acupoint efficacy with Five Element acupuncture [2].

It can be said that a local acupoint directs the treatment strategy to the affected energetic functional sphere or zone (in internal energetic disturbances), while the distal acupoints serve to determine the nature of the energetic manipulation (such as tonification, dispersal, warming, cooling, harmonizing) [14]. The combination of local and distal acupoints forms a pattern of treatment that resembles the pattern of the disharmony being treated. In its most sophisticated application, a treatment pattern and its effects will confirm the diagnosis of the pattern of disharmony. In order to have more effective acupuncture treatment results for other diseases, combination methods that cover local, special and distal acupoints should be used to increase efficacy and serve as tonification or sedation for imbalances. More effective treatment when using Saam acupuncture could be achieved by using it as a root treatment to correct imbalances, while local acupoints, special acupoints and symptomatic treatments are applied as branch treatment to correct interruptions in proper stimulation techniques [4].

\subsection{Promotion and Control Cycles Leading to the Disease Type of Five Evils}

The 50th issue of Nan-Ching explains the pathology of the Five Evils [15] [16] and the 69th issue of Nan-Ching describes the treatment for these evils. This method consists of, in a broad sense, the method of "tonificationsedation between deficiency and excess" as well as the method of "tonification-sedation between coldness and heat".

Among the illnesses are the Depletion, Repletion, Destroyer, Weakness, and Regular Evils, and distinguished as thus:

1) Those (illnesses) coming from behind represent a Depletion Evil;

2) Those coming from ahead represent a Repletion Evil;

3) Those coming from what cannot be overcome represent a Destroyer Evil;

4) Those coming from what can be overcome represent a Weakness Evil;

5) If the respective depot is afflicted from within itself, it represents a Regular Evil.

In case of depletion, it should be filled, while in case of repletion, it should be drained. When neither repletion nor depletion is present, the illness should be removed from the conduits, meaning that in the case of depletion, one should fill the respective (conduit's) mother, and in case of repletion, one should drain the respective (conduit's) child. One must fill first and then drain afterward (Table 2).

One characteristic of Saam acupuncture is the addition of the control (governor) cycle-consisting of grandmother and grandchild elements - to the promotion cycle-consisting of mother and son elements. This method includes a new concept of the "governor Meridian" in the application of the law of the Five Elements based on the inter-destructive cycle.

Wang Sheng Xiu Qiu Si is another concept of the Five Elements [17]. Wang Sheng Xiu is a family and in a promotion cycle of the Five Elements, while Qiu Si is an enemy and in a control cycle of the Five Elements. In promoting the cycle of the Five Elements, a reinforcing method can be done while the control cycle of the Five 
Table 2. Relationship of Five Elements and Five Evils.

\begin{tabular}{cccccc}
\hline Relationship & Mother & Self & Child & Enemy whom I control & Enemy who controls me \\
Five Evil & Depletion & Regular & Repletion & Weakness & Destroyer \\
$\begin{array}{c}\text { Wang Sheng } \\
\begin{array}{c}\text { Principle for } \\
\text { treatment }\end{array}\end{array}$ & $\begin{array}{c}\text { Tonify the mother } \\
\text { for deficiency }\end{array}$ & $\begin{array}{c}\text { Self-tonify or } \\
\text { self-sedate }\end{array}$ & $\begin{array}{c}\text { Sedate the child for } \\
\text { excess }\end{array}$ & $\begin{array}{c}\text { Sedate the enemy for } \\
\text { deficiency }\end{array}$ & $\begin{array}{c}\text { Tonify the enemy for } \\
\text { excess, sedate for deficiency }\end{array}$ \\
\hline
\end{tabular}

Elements require a reducing method. It is meaningful that each Element promotes like a family in the promoting cycle while each Element reduces like an enemy.

Ross [6] calls acupoint selection of tonification and sedation among its own Meridians "Within Element treatments" and acupoint selection of tonification and sedation among other Meridians "Between Element treatments". This selection, in what is called the Four Needle method, is based on the theory of promotion cycle of the Five Elements. He also explains the Eight Needle method which selects another acupoint based on the theory of the inhibition cycle of the Five Elements, in addition to the Four Needle method. He did not explain the originality of its treatments, but the selection is the same as Saam acupuncture.

\subsection{The Six Energy Characteristics of Three Yin and Three Yang}

To achieve a comprehensive understanding of the energy controlled by Meridians, both the Five Elements and Six Energy characteristics must be considered. The characteristic energy controlled by the Meridian is determined not only by the Five Elements, but also by the Six Energy attributes. Six Energies are the subdivision of Yin and Yang energies into three Yin and three Yang, namely Jueyin ("absolute Yin"), Shaoyin ("lesser Yin"), Taiyin ("greater Yin"), Shaoyang ("lesser Yang”), Yangming ("brilliant Yang”) and Taiyang ("greater Yang”). They correspond to Wind, King Fire, Earth, Premier Fire, Metal and Water traits respectively. These Six Energies are subdivided into 12 energies mainly controlled by 12 Meridians that function as energy combined with the Five Elements energy in Meridians located in either the upper or lower extremities. For example, although both the Gall Bladder (GB) and Liver (LR) Meridian energies are Wood, GB energy is Shaoyang while LR energy is Jueyin from the Six Energy perspective. The LR Meridian energy is Jueyin combined with Wood, while the Pericardium (PC) Meridian energy is Jueyin combined with Fire (Premier Fire).

\subsection{Five Elements}

Most illness and disturbances are rooted in interruptions or imbalances in a network of Meridians. Interruptions comprise stagnation and irregularities, and imbalances are deficiency and excess [6].

A typical diagnosis in Saam acupuncture is one of four imbalances of a Meridian energy, which are deficiency, excess, cold or heat. A physician should first find out the Meridian in which the core imbalance of energy is located, and then determine which type the imbalance belongs to. For example, when the energy of Shaoyang, which is expressed as GB or Triple Energizer (TE) Meridian energy, is excessive it may be expressed as a dogmatic personality, sensitive mind or an inclination to give advice to others. When deficient, this energy may be expressed as an atrocious personality, cold mind or a sense of inferiority. More specifically, the energy of GB is associated with centering energy, bright light, aggressiveness, activeness, braveness, dignity or an arrogant attitude, and with energy often compared to a wind power plant or a thunderbolt [18].

The scope of Saam acupuncture can be extensive because of its diverse laws, such as the promotion and inhibition (control) cycle among the Five Elements and its connected Meridians. A Meridian consists of three parts, that is, arm or foot, the more or less of Yin and Yang, and one of Six Organs (Zang) and Six Bowels (Fu). When applying the theory of Yin-Yang and the Five Elements, the essence of what we are after is the energetic movements of disharmonies which are fleeting. The Eight Guiding Criteria make it possible to systematically analyze all data that are gathered in a consistent manner, and thus measure a patient's pattern of disharmony against the major patterns of Chinese medical pathology [14].

\section{Method of Acupuncture Treatment}

The Five Shu points distal to the elbow and knee correspond to the Five Elements and have been used in clinical 
treatments depending on each acupoint's different effects and indication. The combination of Five Shu Points used in Saam acupuncture includes two tonification points and two sedation points. The former, which correspond to the "mother element" according to the law of the Five Elements, tonify the energy of the corresponding channel and Organ. Therefore, the tonification points are needled with stimulation by the tonifying method in Meridian or Organ deficiency conditions.

Each of the 12 Meridians can be subdivided into four types—deficiency, excess, cold, heat-resulting in 48 basic pre-established acupuncture prescriptions, each of which are selections of the Five Shu points. Each Five Shu point corresponds to one of the Five Elements. Traditional use of the Five Shu points incorporates the promotion cycle relationship of the Five Elements where, for example, Wood nourishes Fire and Fire nourishes Earth. As an example, an energy deficiency of the Heart (HT) Meridian (i.e. Fire Meridian) may be corrected by needling on the Wood acupoint (HT9) of the Meridian with a tonification method, based on the above theoretical premise. In Saam acupuncture, acupoints are also selected using the control cycle relationship of the Five Elements as well. For example, Water suppresses Fire and Fire suppresses Metal. To correct the example of Fire deficiency, the Water acupoint (HT3) of the Fire Meridian should be sedated. Saam acupuncture practice would also simultaneously modulate other relative Meridians selected by the theory of the promotion or control cycle relationships, which is necessary for whole-body balance. In the above example, the Wood acupoint of the relevant Wood Meridian (LR1) should be needled using the tonification method, and the Water acupoint of the relevant Water Meridian (KI10) should be needled using the sedation method.

In deficiency condition, tonify the Mother Element. One should select the Mother Element Five Shu point on the Meridian in question for tonification. For instance, in the case of Lung Meridian deficiency, the Mother Element of the Lung is Earth (Earth is the Mother of Metal), and thus the tonification point of the Lung Meridian would therefore be the Earth point of the Lung Meridian, namely LU9 [7]. In excesses situation, the Child Element on the Meridian in question should be sedated. In the case of the Lung Meridian, the Child Element of the Lung is Water (Water is the Child of Metal), and thus the sedation point of the Lung Meridian would be the Water point on the Lung Meridian, namely LU5. The same principle holds true for the other eleven regular Meridians. The four needle method [6] consists of the Within-Reinforce-Tonification point and the Within-ReduceSedation point, which are tonification and sedation of Gao-Wu [7], as well as the Between-Reinforce-Element point and the Between-Reduce-Element point, which are tonification and sedation among other Meridians. They are used in Saam acupuncture in the same way.

Saam acupuncture makes use of the tonification and sedation points along the promotion cycle as well as points along the control cycle in cases of deficiency and excess; and along the fire and water Meridians in cases of heat and coldness. The basic rules, based on the writings of Nan-Ching [15] [16], are those of the promotion and control cycle relationships. In the case of any Meridian's insufficiency (weakness), the points of its mother and its own Meridians should be tonified and the points of its governor and its own Meridians should be sedated. In the case of any Meridian's excessiveness (fullness), the points of its governor and its own Meridians should be tonified and the points of its son and its own Meridians should be sedated [14].

\subsection{Example in Deficiencies}

1) Needle 1: Tonify the deficient Meridian at its mother (tonification) point. With a Lung Meridian deficiency, tonify the Earth point of the Lung Meridian, LU6.

2) Needle 2: Tonify the master point on the Mother Element Meridian. With a Lung Meridian deficiency, the Mother of Metal is Earth, hence the Mother of Lung is Spleen, and the master (Earth) point of the Spleen (Earth) Meridian, SP3, should be tonified.

3) Needle 3: Sedate the Controller Element on the affected Meridian. With a Lung Meridian deficiency, the Controller of Metal is Fire, so the Fire point on the affected Meridian, LU10, should be sedated.

4) Needle 4: Sedate the master point on the Controller Element Meridian. With a Lung Meridian deficiency, the Controller Element is Fire, hence the Controller of Lung is Heart, and the master (Fire) point of the Heart (Fire) Meridian, HT8, should be sedated.

\subsection{Examples in Excesses}

1) Needle 1: Sedate the affected Meridian at its Child Element point. For Liver excess, sedate the Fire (Child) point of the Liver Meridian, LR2. 
2) Needle 2: Sedate the master on the Child Element Meridian. For Liver excess, the Child of Wood is Fire; hence the Child of Liver is Heart. The master (Fire) point of the Heart, HT8, should be sedated.

3) Needle 3: Tonify the Controller Element on the affected Meridian. For Liver excess, the Controller of Wood is Metal, so the Metal point on the affected Meridian, LR4, should be tonified.

4) Needle 4: Tonify the master point on the Controller Element Meridian. With Liver excess, the Controller Element is Metal, hence the Controller of Liver is Lung, and the master (Metal) point of the Lung (Metal), LU8, should be tonified.

To summarize the principle for deficiency conditions [19]: Tonify the first two needles-tonify the Mother Element Five Shu point on the affected Meridian, and then tonify the master point on the Mother Element Meridian; Sedate the second two needles_-sedate the Controller Element Five Shu points on the affected Meridian, and then sedate the master point on the Controller Element Meridian.

For example, if the Stomach is believed to be hypounderactive: (1) for Fire tonification, Stomach Meridian-Fire point ST41, Small Intestine Meridian-Fire point SI5; (2) for Wood sedation, Stomach Meridian-Wood point ST43, Gall Bladder Meridian-Wood point GB41 should be used. The other Meridians follow the same rule, as described above.

To summarize the principle for excess conditions: Sedate the first two needles—sedate the Child Element Five Shu points on the affected Meridian, and then sedate the master point on the Child Element Meridian; Tonify the second two needles-tonify the Controller Element Five Shu points on the affected Meridian, and then tonify the master point on the Controller Element Meridian.

\subsection{Controller Element Meridian}

For example, if the stomach is believed to be overactive: (1) for Metal (son) sedation, Stomach Meridian-Metal point ST45, Large Intestine Meridian-Metal point LI1; (2) for Wood tonification, ST43 and GB41 should be used. The Gall Bladder Meridian is opposed to the Stomach Meridian by the control (destructive) cycle of the law of the Five Elements (the governor Meridian), so the Wood point of Gall Bladder Meridian, GB41, destroys the element of Earth within the self-Meridian. The other Meridians follow the same rule described above (Table 3).

Some kinds of diseases are caused by the imbalance between heat and coldness in the body and the corresponding treatments consist of using the Water and Fire Element Five Shu points along the Meridian in question, the Water Element Meridian, and the Fire Element Meridian. For a heat symptom, the Water point of the self-

Table 3. Saam acupuncture method for symptoms of deficiency and excess.

\begin{tabular}{|c|c|c|c|c|c|c|c|c|}
\hline \multirow{3}{*}{$\begin{array}{c}\text { Meridian } \\
\text { Lung }\end{array}$} & \multicolumn{4}{|c|}{ Deficiency (tonification) } & \multicolumn{4}{|c|}{ Excess (sedation) } \\
\hline & \multicolumn{2}{|c|}{ Tonify } & \multicolumn{2}{|c|}{ Sedate } & \multicolumn{2}{|c|}{ Tonify } & \multicolumn{2}{|c|}{ Sedate } \\
\hline & SP3 & LU9 & HT8 & LU10 & HT8 & LU10 & KI10 & LU5 \\
\hline Large Intestine & ST36 & LI11 & SI5 & LI5 & SI5 & LI5 & BL66 & LI2 \\
\hline Stomach & SI5 & ST41 & GB41 & ST43 & GB41 & ST43 & LI1 & ST45 \\
\hline Spleen & HT8 & SP2 & LR1 & SP1 & LR1 & SP1 & LU8 & SP5 \\
\hline Heart & LR1 & HT9 & KI19 & НT3 & KI19 & НT3 & SP3 & HT7 \\
\hline Small Intestine & GB41 & SI3 & BL66 & $\mathrm{SI} 2$ & BL66 & $\mathrm{SI} 2$ & ST36 & SI8 \\
\hline Bladder & LI1 & BL67 & ST36 & BL54 & ST36 & BL54 & GB41 & BL65 \\
\hline Kidney & LU8 & KI7 & SP3 & KI3 & SP3 & KI3 & LR1 & $\mathrm{KI} 1$ \\
\hline Pericardium & LR1 & PC9 & KI10 & PC3 & KI10 & PC3 & SP3 & PC7 \\
\hline Triple Energizer & GB41 & TE3 & BL66 & TE2 & BL66 & TE2 & ST36 & TE10 \\
\hline Gall Bladder & BL66 & GB43 & LI1 & GB44 & LI1 & GB44 & SI5 & GB38 \\
\hline Liver & KI10 & LR8 & LU8 & LR4 & LU8 & LR4 & HT8 & LR2 \\
\hline
\end{tabular}


Meridian and that of the Water Element Meridian are selected to tonify Water, as well as the Fire points of the self and Fire Element Meridians selected to sedate Fire. For a cold symptom, the Fire points of the self and Fire Element Meridians are selected to tonify Fire, as well as the Water point of the self-Meridian and that of the Water Meridians selected to sedate coldness.

In the case of cold symptoms in the Lung, the coldness symptom can be balanced by both the tonification of heat and sedation of coldness. Accordingly, the Fire points, LU10 of the self-Meridian and HT8 of the Fire Element Meridian, should be tonified to warm "coldness." Conversely, the Water points, LU5 of the self-Meridian and KI10 of the Water Element Meridian, should be repressed. The other Meridians follow the same rules described above.

In the case of heat symptoms in the Lung, the Fire symptom can be controlled by Water, and the Water points, LU5 of the self-Meridian and KI10 of the Water Element Meridian should be tonified. Conversely, the Fire points, LI10 of the self-Meridian and HT8 of the Fire Element Meridian, should be repressed. The other Meridians follow the same rule described above (Table 4).

\section{Method of Tonification and Sedation}

Saam acupuncture is applied with stimulation techniques such as respiratory, rotational and directional methods. Each stimulation results in tonification or sedation. Tonification and sedation occur depending upon clockwise or counter-clockwise rotations in accordance with direction of Meridians. Tonification occurs following the insertion of a needle as the patient exhales and removal at inhalation. Sedation occurs on insertion of the needle as the patient inhales and removal on exhalation. Tonification occurs when the needle is inserted in the inclined position following the direction of the flow of $Q i$ in the Meridian. By contrast, sedation occurs following insertion of the needle in the inclined position in the opposite direction to the flow of Qi in the Meridian [2]. In theory, the use of acupuncture stimulation may be possible in treatment but strong technical movements on acupoints located on shallow body surfaces, such as between finger tips and elbow or tips of toes and knee, may be hard for the practitioner to access. Clinically it is recommended that practitioner do not use strong stimulations. A simple and weak stimulation seems to be enough to gain the acupuncture effect.

\section{Diagnosis Method}

The scope of Saam acupuncture can be extensive because of its diverse laws, such as the promotion and inhibi-

Table 4. Saam Acupuncture Method for Symptoms of Cold and Heat.

\begin{tabular}{|c|c|c|c|c|c|c|c|c|}
\hline \multirow{4}{*}{$\begin{array}{c}\text { Meridian } \\
\text { Lung }\end{array}$} & \multicolumn{8}{|c|}{ Symptoms } \\
\hline & \multicolumn{4}{|c|}{ Cold } & \multicolumn{4}{|c|}{ Heat } \\
\hline & \multicolumn{2}{|c|}{ Tonify } & \multicolumn{2}{|c|}{ Sedate } & \multicolumn{2}{|c|}{ Tonify } & \multicolumn{2}{|c|}{ Sedate } \\
\hline & HT8 & LU10 & LU5 & KI10 & LU5 & KI10 & SP3 & LU9 \\
\hline Large Intestine & SI5 & ST41 & LI2 & BL66 & LI2 & BL66 & SI5 & ST41 \\
\hline Stomach & ST41 & SI5 & ST44 & BL66 & ST44 & BL66 & ST36 & BL54 \\
\hline Spleen & SP2 & HT8 & SP9 & KI10 & SP9 & KI10 & SP3 & KI3 \\
\hline Heart & HT8 & KI2 & HT3 & KI10 & HT3 & KI10 & HT8 & KI2 \\
\hline Small Intestine & SI5 & BL60 & SI2 & BL66 & SI2 & BL66 & SI8 & ST36 \\
\hline Bladder & SI5 & BL60 & SI2 & BL66 & SI2 & BL66 & ST36 & BL54 \\
\hline Kidney & HT8 & $\mathrm{KI} 2$ & KI10 & HT3 & KI10 & HT3 & SP3 & KI3 \\
\hline Pericardium & HT8 & PC8 & PC3 & НT3 & PC3 & HT3 & SP3 & PC7 \\
\hline Triple Energizer & TE6 & BL60 & TE2 & BL66 & TE2 & BL66 & TE6 & BL60 \\
\hline Gall Bladder & GB38 & SI5 & GB43 & BL66 & GB43 & BL66 & BL54 & GB34 \\
\hline Liver & LR2 & HT8 & KI10 & LR8 & KI10 & LR8 & LR3 & SP3 \\
\hline
\end{tabular}


tion (control) cycles among the Five Elements and their corresponding Meridians. A Meridian consists of three parts; that are, upper extremity or lower extremity, more or less of Yin and Yang, and one of Six Organs (Zangs including Pericardium) and Six Bowels (Fus). A total of 24 deficiency and excess symptoms exist across the Six Organs and Six Bowels, but theories related to these symptoms are too arbitrary to assist in making a diagnosis. Although there are currently many clinical books on Saam acupuncture in Korea, a definitive general standard for the selection of symptom patterns has not yet been established. The general use of Saam acupuncture has previously been discussed in 1985 [20]. The method can be used mainly from a theoretical viewpoint of the Organs, etiology of disease, as well as the more or less of the three Yang and three Yin. For example, in the case of knee pain, one can use Liver-tonification (mostly deficiency condition) because, according to the theory of the Organs, the Liver controls the muscles and joints. Secondly, in the case of headache due to increasing LiverYang, one may use Liver-sedation (mostly excess condition) given the etiology of the disease. Furthermore, in the case of backache due to dampness, we can use either Spleen-sedation (excess condition) to sedate dampness or Yangming-tonification (deficiency of Large Intestine or Stomach) to tonify dryness from the principle of the more or less of the three Yang and three Yin.

The above three viewpoints cannot fully explain Saam acupuncture, so a common differential diagnosis shared by many acupuncturists needs to be determined. Lee [21] was the first to propose a differential diagnosis of deficiency and excess by simultaneously comparing the six pulses of both wrists in the 1960s. However, his diagnosis was only focused on deficiency and excess, neglecting coldness and heat. Although he said that irregular forms of treatments were the manifestation of subtle changes in diseases, signs and symptoms are needed to support his assertions. Lee's comparative pulse diagnosis is very original in that it attempted to determine different symptoms, but a diagnosis made only using the pulse method is difficult. Comparative pulse diagnosis can therefore be an important guide in using Saam acupuncture, despite its weakness.

Kim [22] stated that he proposed the diagnosis method called "Saam Five Elements acupuncture of disease symptoms," which differentiates symptoms by simultaneously comparing the pulse, as he found that the original Saam acupuncture could not cope with changes in diseases. He asserted that a floating pulse showed Yang excess and Yin deficiency, while a deep pulse demonstrated Yin excess and Yang deficiency on the basis that the medial pulse is neither floating nor deep, and is the manifestation of health. Kim also proposed, from his clinical experience, that more focus needs to be placed on the symptoms of coldness and heat rather than the symptoms of deficiency and excess when differentiating these two symptom types. This can be achieved by comparing both the right and the left Guan pulses. If the right Guan pulse is weaker than the left the symptom is related to coldness and heat, while if the left Guan pulse is weaker than the right Guan pulse the symptom is related to either deficiency or excess. For example, looking at the symptom of either coldness or heat, if the pulse is floating, the main symptom is Yang excess and Yin deficiency. The treatment is then to first tonify the fire within the Yin Meridian and then to sedate the coldness within the Yang Meridian. In the heat symptom, if the pulse is deep, the main symptom is Yin excess and Yang deficiency. The treatment is to first tonify the coldness within the Yang Meridian and then to sedate the heat within the Yin Meridian.

The method used by Kim is more detailed than that of Lee, in that Kim used differentiation of coldness \& heat symptom type and deficiency \& excess symptom type, and set the criterion for Yin-Yang balance by standardizing medial pulse. Kim's theory is still contentious and not fully accepted as a standard of general treatment as it is difficult to decide differential symptoms by pulse diagnosis only. The merit of Kim's treatment is that it contributed greatly to the objective use of Saam acupuncture [7].

Seem [11] also believes that treatments using the four-needle technique are very powerful, and should only be pursued when a strong energetic manipulation is required, and only when one is certain of the primary diagnosis of the affected Five Elements or Phases. This recommendation is because the four-needle technique tonifies twice, then sedates twice, leading to a very concentrated tonifying or sedating action on the affected Five Elements and Meridian. If one is not certain of the primary affected Element, it is far more conservative to use more gentle tonification and sedation acupoints.

Generally speaking, Saam acupuncture primarily focuses on deficiency and excess symptoms rather than coldness and heat symptoms, although the relationship between the deficiency and excess symptom type and coldness and heat symptom type needs to be studied in detail in order to create reasonable guidelines for effective treatments. As there are many different ways to select Five Shu points from the various opinions concerning Five Element acupuncture, we need to determine detailed guidelines for patterns of symptoms in the future. 


\section{Practice of Saam Acupuncture}

Saam acupuncture is primarily focused on Five Shu points, which contradicts other acupuncture methods. For example, backache can be caused by disturbances of the Bladder Meridian, so one can use tonification or sedation of the Bladder Meridian depending upon deficiency or excess. Backache can also be caused by weakness of the Kidney Meridian, which one can then treat by using tonification of the Kidney Meridian. Finally, backache is caused due to excessive dampness of the Spleen, which can be diagnosed in obese people. One can then use tonification of the dry Large Intestine Meridian or Stomach Meridian in order to dry out dampness. Sedation of the damp Spleen Meridian can also be used on rare occasion. The selection of tonification or sedation depends wholly upon the diagnosing practitioner.

Backache is generally associated with Kidney or Bladder functions. The Governor vessel and Gallbladder Meridian, with little connection to other Meridians, can also be involved in backache. Saam acupuncture treats backache using a novel approach. It relates backache to the malfunction of connected Organs (i.e. the Bladder, Kidney and Gall Bladder) to the imbalance of Yin and Yang (Six Qi) while primarily using Five Shu acupuncture points. It is a wonder how the combination of the acupoints distal to the painful region and located beneath the elbow and knee joints can treat diseases such as backache, shoulder pain, and whatnot. This wonder may be understood using Yun-Tao-Mao's neurobiological fact: as the limbs below the elbows and knees occupy larger areas in the sensory gyrus in the brain and the acupoints below the elbows and knees contain more sensory receptors, needling stimulation to these points may induce a greater reaction and activity in the brain. This principle clearly supports the concept of using certain acupoints below the elbows and knees, such as the Five Shu points, as diagnosis and treatment points during acupuncture treatment.

In cases of interruption, acupoints are selected based on local and special effects in correlation with efficacy. Five Shu points are selected for either deficiency or excess conditions to tonify or sedate in cases of imbalance. Overall acupuncture treatment method that covers the treatment of interruptions and imbalances of illnesses should be selected. For instance, more effective treatment result may be achieved if we use Saam acupuncture as a "root treatment" to correct imbalances but then combine it with Meridian style acupuncture, channel therapy acupuncture (which uses local and distal acupoints), special acupuncture points, or symptomatic treatment Meridian therapy, as "branch treatments", to correct interruptions in proper stimulation technique.

The original form of Saam acupuncture can be used from the perspective of the Five Organs, disease etiology, imbalanced Yin and Yang, the mind-only theory and the comparing-pulse diagnoses such as Lee [21] and Kim [22].

\section{Saam Acupuncture in Research}

It was reported that treatment following Saam acupuncture theory produced a positive effect on the dysarthria symptom of stroke patients, which was more prominent than the effects noted from using body acupuncture common in Korean acupuncture and traditional Chinese medicine [23]. It was also reported that acupuncture following Saam acupuncture treatment combined with conventional body acupuncture produced more pronounced results than those of body acupuncture alone [24].

One recent study [25] reported that Saam acupuncture may attenuate the imbalance between sympathetic and parasympathetic activities induced by night-shift work in nurses. Hwang et al assessed the effects of Saam acupuncture on the autonomic nervous system in night-shift nurses using power-spectral heart-rate variability (HRV) analysis. This study had a $2 \times 4$ cross-over design with a series of six $(n=1)$ controlled trials. Six night-shift nurses were randomly divided into two groups, and each nurse received four acupuncture treatments on the third day of night-shift work. One group started with Saam acupuncture (Gall Bladder-tonification), while the other started with sham acupuncture. Saam acupuncture and sham acupuncture were applied in turn. HRV was measured before and after treatment. For statistical analysis, the results of the two groups were combined, and a Bayesian model was used to compare the changes in HRV values before and after treatment, between Saam and sham acupuncture. As the ratio of low-to high-frequency power (LF/HF) for HRV increased on the third day of night-shift work in the pilot study, HRV measurements were made on the third day. Compared with sham acupuncture, Saam acupuncture reduced sympathetic activity; the overall median treatment effect estimate in LF normalized units decreased by -17.4 (confidence interval (CI): $-26.67,-8.725$ ) and that for LF/HF decreased by -1.691 (CI: $-3.222,-0.3789$ ). The overall median treatment effect estimate in HF normalized units increased by 17.41 (CI: 6.393, 27.13) with Saam acupuncture, suggesting an increase in parasympathetic activity. 


\section{Taegeuk Acupuncture}

Taegeuk acupuncture is based on Sasang constitutional medicine, which makes the Heart the Central Ultimate, or taichi ("taegeuk"), of the Organs and classifies the body into four types by the relative size of the Organs. It decides the constitution first and tonifies or sedates the source point of the relevant Organ according to the size of the Organs. For example, Tae-yang (Greater Yang) constitution belongs to Metal, Tae-eum (Greater Yin) to Wood, So-yang (Lesser Yang) to Fire, and So-eum (Lesser Yin) to Water, and thus the Fire, Metal, Water, and Earth acupoints (HT8, HT4, HT3, HT7) of the Heart Meridian are tonified, for they are of the Five Elements that control the original dominant Organs. By doing so it compensates for the loss of balance, and can further balance the power of Organs by depleting the source point of the relevant Meridian according to the size of the other Organs, thus helping the patient get over his or her own disease by him or herself.

Sasang constitutional medicine describes four constitutions-Tae-yang, Tae-eum, So-yang and So-eumwhich indicate constitutional energy discrepancies among major Organs. According to the Sasang constitutional theory, an aggravated energy discrepancy among major Organ energies is the underlying causative imbalance of superficial pathologic conditions [4] [26]. Lee Je-Ma, founder of the Sasang constitutional medicine, prescribed different combinations of herbs for his patients based on their respective constitutions, even if the patients came to seek him with apparently similar diseases. Rather than dealing with thousands of different diseases, practitioners could deliver four simple types of treatment for diverse illnesses aimed at recovering the energy balance of the constitution. Lee Je-Ma's original system of Sasang constitutional medicine lacked an acupuncture treatment system for the four constitutions.

Taegeuk acupuncture is originated by Lee Byeong-Haeng, as a response to Lee Je-Ma's testament that stated, "A doctor needled LI4 of a patient of So-eum constitution suffering dysarthria from stroke and the effect was astonishing that there may be other diseases that are better cured by acupuncture than medicine. Acupoints may be applied to the four constitutions as well; therefore further research is called for in the future". LI4 is the source point of the Large Intestine Meridian, and theoretically in Sasang constitution the Large Intestine is substituted for Kidney because of the principle that the Kidney should not be sedated. The source point is sedated for Kidney is the large Organ in So-eum constitution.

Taegeuk acupuncture provides acupuncture prescriptions for the correction of the underlying energy discrepancies that are assumed in Sasang constitutional medicine. Lee Byeong-Haeng suggested that observation of reactions to acupuncture stimulation of the Heart Meridian acupoints might be helpful in determining the type of constitution that the patient belongs to, because Heart energy plays a central role in controlling other Organs in the system of Sasang constitutional medicine [4].

For example, the constitutional characteristics of the Tae-yang constitution come from the discrepancy between a large Lung and a small Liver energy. According to the Taegeuk acupuncture theory, Tae-yang constitution is considered to be in a state of Metal excess, which may be regulated by stimulating HT8, the Fire point of the Heart Meridian. Fire suppresses Metal from the perspective of the control cycle relationships of the Five Elements. Similarly, people with the Tae-eum constitution have a large Liver and small Lung energy, and are considered to be in a state of Wood excess, which can be regulated by stimulating HT4, the Metal point of the Heart Meridian.

Kim [27] testifies that in his experience the duration of treatment lasted in average three to four months, and some diseases took six to seven months to cure.

Lee Byeong-Haeng used the rotation method and numbers method to tonify and sedate. Kim added respiratory method to prevent fainting during acupuncture intervention. To tonify, place needle while the patient inhales and rotate clockwise for nine times or the multiple of nine times. To sedate place needle while the patient exhales and rotate counter-clockwise for six times or the multiple of six times. Rotate until deqi is attained.

The judgment of the constitution may be the most important step in practicing Taegeuk acupuncture. It is done by tonifying the controlling Five Elements acupoint of the Heart Meridian and tonifying or sedating the source point of the small and large Organs' Meridians of an assumed constitution. After placing those three needles, press the solar plexus to confirm the constitution by the tenderness response. When the needles placed are in accord with the patient's constitution the tenderness is gone about $60 \%-70 \%$, and when the acupoints are not relevant with the patient's constitution they reply about $20 \%-30 \%$ of the tenderness is gone. It is best to do the test 1 - 2 minutes after practicing Taegeuk acupuncture.

The symptoms for which Taegeuk acupuncture is effective are listed below by Lee. The exemplified treat- 
ments are for patients with So-yang constitution.

1. A stroke patient with hemiplegia can move his or her limbs when HT3 is tonified.

2. Tinnitus disappears at the site.

3. Headache, stomachache and numbness of hands and feet are cured at the site.

4. Hiccupping stops short.

5. It is hard to confirm the cure of stomach cancer but the pain disappears at the site.

6. Eyesight becomes bright.

7. Fatigue is recovered at the site.

8. Myalgia is reduced.

9. Low back pain is cured at the site.

Kim also summarizes his clinical experience and introduces the response of patients after Taegeuk acupuncture.

1. Taegeuk acupuncture is effective for psychogenic disease with symptoms such as palpitation, forgetfulness, insomnia, anxiety, impatience, chest discomfort, shortness of breath, headache, dizziness, and nausea. They relate to disease such as depression, chronic insomnia, aphasia, tic disorder and deficiency of Heart, Gall Bladder and Spleen.

2. Taegeuk acupuncture is effective for illnesses caused by blood circulation disorder such as blurry eyesight, pallor, headache, dizziness, stiff neck, menstrual pain, morning edema, small joint pain, etc.

3. Taegeuk acupuncture is effective for incurable diseases such as hand tremor, shaky head, facial spasm, convulsion of unknown reason, and for diseases for which standard acupuncture worsens the symptoms.

4. Taegeuk acupuncture can be used alongside standard acupuncture for:

1) Tinnitus or sudden deafness by needling TE21, SI19, TE17 of the affected side;

2) Trigeminal neuralgia by needling ST4-ST6, ST7-TE17, GB14-TE23 (electro-acupuncture) of the affected side;

3) Bell's palsy by needling ST4-ST6, ST7-TE17, GB14-TE23 (electro-acupuncture) of the affected side;

4) Unhealed ankle sprain after 3-6 months by using standard acupuncture following the Meridian of the affected side.

In cases of patients with marked solar plexus tenderness, Taegeuk acupuncture is practiced after the diagnosis of the constitution, regardless of the chief complaint whether it is stiff neck, lower back pain or knee joint pain. The following reaction usually results.

1) The mind is calmed and head becomes clear;

2) Chest discomfort disappears;

3) Vision becomes bright;

4) Digestion is boosted, and one feels hunger;

5) One becomes drowsy;

6) The acupuncture treatment site is painful;

7) One does not notice any difference.

The above 1 - 7 reactions are those of the patients right after one treatment session of Taegeuk acupuncture. After 1 - 2 days of the first treatment, the reactions are as follows:

1) Sleeps well at night after acupuncture treatment;

2) Body feels lighter;

3) Fatigue seems to be less;

4) Chronic and recurrent joint pain such as neck stiffness, knee pain and backache seems to be relieved;

5) Myalgia and spasm seem to be improved;

6) Still not sure of any difference.

In one recent study [28] Taegeuk acupuncture had potential as an effective means of stabilizing mental stressinduced imbalance of autonomic nervous system for So-yang constitution. In this study eight women diagnosed as So-yang constitution participated in a study on reducing mental stress assessed by heart rate variability. They were randomly divided into group A and group B. Each participant went through 3 sessions every week with 1 week of washout period in between each session. HRV was measured three times at every session; at baseline, after administering mentally stressful circumstances and after applying either one of simple rest, So-yang Taegeuk acupuncture or So-eum Taegeuk acupuncture. This study was designed as a crossover clinical trial. After same initial simple resting session for both groups at week 1 , acupuncture for group A were executed in the or- 
der of So-yang Taegeuk acupuncture and So-eum Taegeuk acupuncture at week 2 and 3 respectively, with acupuncture for group B conducted in reverse order. The simple rest and So-eum Taegeuk acupuncture did not show the significant changes in response to LF(norm) and HF(norm) after stress stimuli. So-yang Taegeuk acupuncture did, however, significantly decrease LF(norm) and increase HF(norm). So-yang Taegeuk acupuncture, compared to So-eum Taegeuk acupuncture, significantly stabilized autonomic nervous system.

\section{References}

[1] Yin, C., Park, H.J., Chae, Y., Ha, E., Park, H.K., Lee, H.S., et al. (2007) Korean Acupuncture: The Individualized and Practical Acupuncture. Neurological Research, 29, 10-15. http://dx.doi.org/10.1179/016164107X172301

[2] Kim, D.-H. and Kim, J.-H. (1993) The Literary Study on the Written Date of and the Background of Sa-Ahm's 5 Element Acupuncture Method. Kyungwon University, Kyungki.

[3] Yin, C.-S. Chae, Y.-B., Koh, H.-G., Lee, H.-J., Chun, S.-I., et al. (2006) Constitutionally Individualized and Practically Integrated Characteristics of Korean Acupuncture. Korean Journal of Acupuncture, 23, 19-28.

[4] Ahn, C.B., Jang, K.J., Yoon, H.M., Kim, C.H., Min, Y.K., Song, C.H., et al. (2010) Sa-Ahm Five Element Acupuncture. Journal of Acupuncture and Meridian Studies, 3, 203-213. http://dx.doi.org/10.1016/S2005-2901(10)60037-4

[5] Lee, T.H. (1996) Essentials of Saam's Acupuncture Theory. Haenglim Publishing, Seoul.

[6] Jeremy, R. (2004) Acupuncture Point Combination. Churchill Livingstone, Edinburgh.

[7] Wu, G. (1980) Gatherings from Eminent Acupuncturists-Zhen Jiu Ju Ying. Shinmunfung Publisher, Taipei.

[8] Heng-pa (1975) The Essence of Sa-Ahm's Acupuncture. Heng-Lim Publisher, Seoul.

[9] Hicks, A.H.J. and Mole, P. (2005) Five Element Constitutional Acupuncture. Churchill Livingstone, Edinburgh, 383384.

[10] Zhang, Y. (1977) Huang-Ti Nei-Ching Su-Men Ling-Shu Ji-Zhu He-Pian. Tai-Lian Guo-Feng Chu-Ban She, Taipei.

[11] Ahn, C.B., Jang, K.J., Yoon, H.M., Kim, C.H., Min, Y.K., Song, C.H., et al. (2009) A Study of the Sa-Ahm Five Element Acupuncture Theory. Journal of Acupuncture and Meridian Studies, 2, 309-320. http://dx.doi.org/10.1016/S2005-2901(09)60074-1

[12] Maciocia, G. (2005) The Foundation of Chinese Medicine. Churchill Livingstone, Edinburgh.

[13] Ma, Y. and Cho, Z. (2005) Biomedical Acupuncture for Pain Management. Churchill Livingstone, Edinburgh.

[14] Seem, M.D. (1991) Acupuncture Energetics. Healing Arts Press, Rochester.

[15] Chang, S. (1912) Chiao-cheng t’u-chu pa-shih-i Nan-Ching. Hung-pao chai shu-chu, Taipei, 6-11.

[16] Unschuld, P.U. (1986) Nan-Ching - The Classic of Difficult Issues. University of California Press, Oakland, 474-617.

[17] Lee, I. (2007) A Study on the Basic Forms and Principles of Saam's 5 Phase of Acupuncture Method. Journal of the Korean Acupuncture and Moxibustion Society, 1, 19-51.

[18] Kim, H.G. (1992) Handbook of Saam Acupuncture. Shinnongbaekcho Press, Seoul.

[19] Kim, D. (2002) Reviewed Sa-Ahm's Acupuncture Treatment with Graphs. So-Gang Pub, Busan.

[20] Ahn, C.B. and Choi, D.Y. (1986) Theoretical Study on Five Element Acupuncture. Dongguk Journal, 5, $287-309$.

[21] Lee, J.W. (1958) The Secret of Saam's Acupuncture Based on Yin-Yang and Five Elements. Institute for Studying Five Element Acupuncture, Busan.

[22] Kim, D.P. (1972) Saam’s Five Element Acupuncture and Its Usages. The Journal of the Korean Oriental Medical Society, Winter Issue, 122-123.

[23] Song, M.S., Kim, Y.H., Jang, S.G., Kim, J.H., Yim, Y.K., Kang, J.H., et al. (2003) Clinical Comparison Studies on 20 Cases of Stroke Patients with Dysarthria by Sa-Am and General Acupuncture. Journal of the Korean Acupuncture and Moxibustion Society, 20, 161-167.

[24] Kim, J.H., Park, E.J., Park, C.H., Cho, M.R., Ryu, C.R. and Chae, W.S. (2002) Comparison of the Improvement of Back Pain and Sciatica between Common Acupuncture Treatment Group and Common Acupuncture with Shin Jong Gyuk of Ohaeng Acupuncture Treatment Group. Journal of the Korean Acupuncture and Moxibustion Society, 19, 84-91.

[25] Hwang, D.S., Kim, H.K., Seo, J.C., Shin, I.H., Kim, D.H. and Kim, Y.S. (2011) Sympathomodulatory Effects of Saam Acupuncture on Heart Rate Variability in Night-Shift-Working Nurses. Complementary Therapies in Medicine, 19, S33-S40. http://dx.doi.org/10.1016/j.ctim.2010.11.001

[26] Kuon, D.W. (1965) A Study of Constitution-Acupuncture. Journal of the International Congress of Acupuncture and Moxibustion, 10, 149-167. 
[27] Kim, J.K. (2011) Clinical Opinion of Taegeuk Acupuncture Treatment by Sasang (4-Type) Constitutional Medicine. Journal of the Korean Acupuncture and Moxibustion Society, 28, 69-73.

[28] Kim, N.S., Kim, S.J., Ryu, H.J., Nam, S.S. and Kim, Y.S. (2012) Effects of Taegeuk Acupuncture on the Autonomic Nervous System by Analyzing Heart Rate Variability in Soyangin. Journal of the Korean Acupuncture and Moxibustion Society, 29, 81-88. 